Research Article

\title{
IDENTIFICATION OF PARKINSON'S DISEASE BY AR MODELLING OF GAIT SIGNALS
}

\author{
$\operatorname{Omer} A K G U N^{1 *}$, Aydin $A K A N^{2}$ \\ Parkinson's is a neurodegenerative disease that, as in the case of other \\ neurodegenerative diseases, has disruptive effects on human mobility. In this \\ study, gait markers were obtained by using sensors under the foot, giving an \\ output proportional to the force. Normal gait markers were compared with \\ those of Parkinson's patients. Thus, individuals with Parkinson's were \\ identified by comparing the impulse model of gait markers obtained from \\ normal individuals with those of Parkinson's patients.
}

Key words: Parkinson, gait markers, impulse model

\section{Introduction}

Disease was identified using the differences between the impulse models of these processes.

One of the fundamental objectives of current clinical research into neurodegenerative diseases is the development of early detection methods and presymptomatic diagnosis of neuronal dysfunction [1] . Several different approaches are being undertaken to identify bioindicators, involving the use of imaging, neurophysiological and cognitive tests, in addition to the latest technologies such as biochemical, proteomic, metabonomic and gene-array profiling of tissue and biofluids taken from patients. Key recent findings in each of these areas are discussed, including the walking function [2] .

In order to be able to walk for long periods of time without tiring, the muscle-skeleton and nervous systems need to be healthy. Brain, spinal cord, peripheral nerves, muscles, bone and joints should work together; joint movements, timing and force of the contractions muscular contractions should be adequate.

In order to move the body forwards, a repetitive series of movements occur in the legs. This movement cycle, which is repeated in a defined sequence, is called the walking cycle.

Gait analysis is the examination of a person's walk, both kinetically (in terms of factors which cause joint movements such as force, pressure, momentum, torque and factors which determine kinematical sizes) and kinematically (in terms of translocation, linear speed, acceleration, and corresponding angular sizes) [3].

Scientific evaluation of human movement goes back to the time of Aristo. In 350 B.C., Aristo found that the joint movements were caused by muscle contraction. A few centuries later, Galen (A.D. 131-201) asserted that nerves governed muscle contraction. During the Renaissance period, Galileo's student Borelli, and Marey, in the 19th century, conducted dynamic studies relating to the human walk. In the late nineteenth century, the photographer Eadweard Muybridge made significant

\footnotetext{
1 Department of Computer Engineering University of Marmara, Istanbul, Turkey(e-mail: oakgun@marmara.edu.tr) (Dhttps://orcid.org/0000-0003-3486-2197

2 Department of Biomedical Engineering University of Izmir Katip Celebi, Istanbul, Turkey (e-mail: aydin.akan@ikcu.edu.tr) (Dhttps://orcid.org/0000-0001-8894-5794
} 
contributions to gait analysis. Braune and Fischer subsequently converted photographic images to numeric variables in 1895 .In the 1930s, Eberhart and Inman repeated these experiments in detail by using a rotating perforated disk in front of the camera lens. Verne Inman and Jacquelin Perry introduced scientific gait analysis as we know it today into clinical practice, with their studies beginning in the 1950s. Since the rapid development of personal computers in the 1980s, walking analysis systems have been developed for clinical practice and are widely used throughout the world [4].

Gait analysis is the numeric evaluation, definition and interpretation of walking . Although many walking problems can be detected with visual examinations conducted by experienced doctors, walking analysis technology is required in order to interpret the problem numerically, to record and subsequently re-evaluate it, and to objectively evaluate the effectiveness of the treatment [5]. In modern walking analysis laboratories, the patient's walk is first evaluated visually and with video recordings. Movement data is then transferred to the computer via transmitter or reflectors attached to appropriate points on the patient's body; additionally, variations in floor reaction force measured when standing on a floor-mounted force platform are also uploaded to the computer. It is also possible to measure dynamic electromyography and energy consumption [6]. Diagnosis is made, based on evaluation of the data and the clinical status of the patient. Previous studies, which come within the scope of this paper on the numerical evaluation of neurodegenerative diseases and gait dynamics, were conducted by Hausdorff et al. [6,7]. Those studies were generally based on analysis relating to gait rhythm [7].

The present study is based on the modeling of gait markers, obtained by using sensors sensitive to the force beneath the foot, using the AR process. Parkinson Disease was identified using the differences between the impulse models of these processes.

\section{Parkinson Disease}

Parkinson Disease (PD) is the second most prevalent neurodegenerative disease worldwide, and is the primary movement disorder in terms of frequency.

Parkinson's is a slow onset clinical outlook, which generally emerges in those over the age of 50 , and which rarely appears under the age of 40 . One of the most distinct symptoms in Parkinson's is the tremor observed in the resting state, which is mostly observed in the hands. This tremor generally has 3-7 beats per second and disappears during purposeful movement and sleep, for example when reaching to pick up an object. This type of tremor observed in Parkinson's patients is one of the most important clinical symptoms in which the electroencephalography (EEG), electromyogram (EMG) interaction is clearly observed [8].

Another important symptom of Parkinson's is the increase in muscle tone . Consequently, there is stiffening in the patient, when the patient attempts a passive movement, a resistance is encountered. For example, when one attempt to open the patient's bent arm opening the elbow joint, the movement becomes more difficult due to the resistance encountered.

Another important symptom is the general slowing of movements. In Parkinson Disease and Parkinsonism, which are the most important diseases of the extrapyramidal system, walking is pathognomonic. Patients walk with a flexed posture, with small and gradually more rapid steps. Patients have difficulty in commencing walking, changing direction and stopping whilst walking; The number of steps per minute does not vary significantly; The step width increases; Hip, knee and foot joint movements are reduced; Arm swing reduces; Opposite direction pelvis and body rotation is 
observed; In the initial contact phase, stepping with the entire foot sole instead of the heel is observed, and turning in the heel deteriorates.

In addition to the above symptoms, patients may display reduction in mimics, talking with a monotonous and low voice and difficulty in sitting down or getting up [9].

In Parkinson Disease, the nerve cells in the substantia nigra, the structure of the brain which produces dopamine, are damaged and reduced. The reason for the damage caused to these cells is still unknown at present. It is evident that this is not a random situation and that it is not due to atherosclerosis, weak blood circulation, or inflammatory or changes of microbial origin. It has been suggested that a deficiency in certain undefined substances, or an unknown toxin may be responsible for this cell damage.

The extensions of these cells are in areas called "striatum" (stripy object). The excreted dopamine adheres to the receptive structures (receptors) found here and transmits information from one nerve cell to another. As sufficient dopamine is not produced in the brain, symptoms of Parkinson Disease emerge which manifest themselves in the form limb tremors in the resting state, muscle rigidity, slowness of movement and posture deformity. Before the symptoms emerge, approximately $60 \%$ of the dopaminergic neurons in substantia nigra and $80 \%$ of the dopamine in the striatum are lost [10].

\section{Application}

This study is based on the kinetically analysis of gait dynamics. Kinetically analysis is the examination of the forces which create movement (floor reaction forces, joint moments, and joint strengths). The only measurable data in kinetically analysis is the floor reaction force vector (FRFV) [11]. FRFV is measured with pressure-sensitive plates, called a force platform, which measure the total force applied on the floor by the foot. Transducers placed on both sides, front, back and inside of the platform measure the components in all three planes of the load placed on the surface of the platform and transfer this data to the computer [12]. Another option, used in the present study, is to record gait markers by placing force sensitive piezoelectric sensors under the foot (Figure 1).

In this way, 60 second long gait markers were recorded. These were converted to 18,000 series at $300 \mathrm{~Hz}$ frequency using an analog-digital converter and transferred to the computer. Gait markers were taken from the left feet of 11 normal individuals and 12 Parkinson patients.

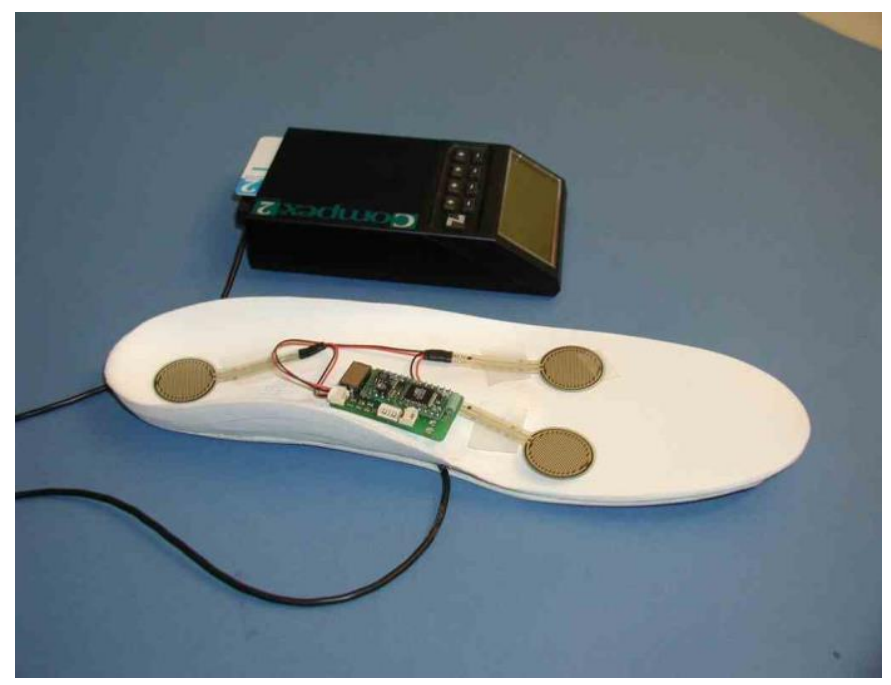

Figure 1. Piezoelectric sensors under the foot. 


\section{AR Modelling of Gait Signals}

Model-based (parametric) methods are based on modeling of the $y(n)$ data series as the output of a linear system characterized by a rational system [13].

A dynamic linear system (Figure 2a)) is defined by unit impulse response [14]. With as input, as output and as noise markers, the system is modeled with unit impulse response. Output expression gives the formula:

$$
y(n)=\sum_{k=1}^{\infty} h(n) \cdot x(n-k)+v(n)
$$

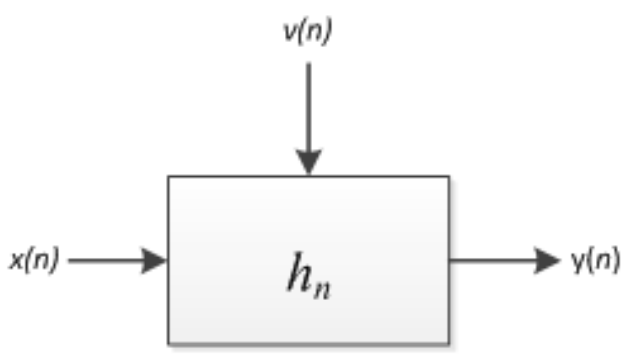

a)

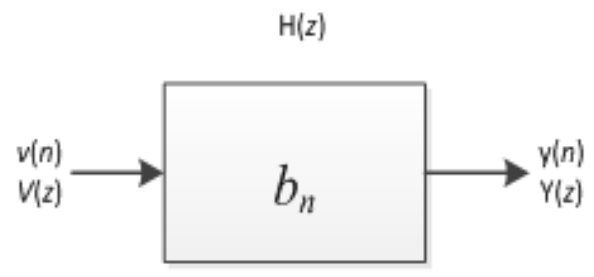

b)

Figure 2. A dynamic linear system.

AR models are used in discrete time systems encountered in practical applications, and in situations when it is necessary to model the system using only the output values as data. In the AR model, the $y(n)$ gait marker's current value and previous values are equal to the total of $y(n-1), y(n-$ $2), \ldots \ldots \ldots, y(n-M)$ finite linear combination and the error term $v(n)$. Therefore, if a $y(n)$ time series provides the difference equation

$$
y(n)=b_{1} \cdot y(n-1)+b_{2} y(n-2)+\ldots \ldots+b_{M} . Y(n-M)+v(n)
$$

This is an AR process of the M. degree. The $b 1, b 2, \ldots \ldots, b M$ constants are AR parameters, whilst $\mathrm{v}(\mathrm{n})$ is a white noise process $[15,16,17]$.

In Figure 3, the system, which is only y(n) output expression, is expressed with the formula:

$$
y(n)=\sum_{k=0}^{M} b_{k} \cdot v(n-k)+v(n) \quad \mathrm{b}_{0}=1
$$

The transfer function of the AR model using $\mathrm{Z}$ transformation will be:

$$
H(z)=\frac{Y(z)}{V(z)}=\frac{1}{\sum_{n=0}^{M} b_{n} \cdot z^{-n}}
$$


The impulse models of normal and Parkinson gait markers modelled with a 4th degree AR process as in Figure 2b) are shown in Figure 3.a),b),c),d).

Table 1. Peak values of impulse models of gait markers.

\begin{tabular}{|c|c|c|}
\hline $\begin{array}{c}\text { Patient } \\
\text { No }\end{array}$ & Normal & Parkinson \\
\hline 1 & 0.0464 & 0.107 \\
\hline 2 & 0.047 & 0.116 \\
\hline 3 & 0.0473 & 0.119 \\
\hline 4 & 0.0405 & 0.112 \\
\hline 5 & 0.149 & 0.137 \\
\hline 6 & 0.0421 & 0.118 \\
\hline 7 & 0.131 & 0.106 \\
\hline 8 & 0.158 & 0.127 \\
\hline 9 & 0.151 & 0.104 \\
\hline 10 & 0.0373 & 0.126 \\
\hline 11 & 0.0441 & 0.128 \\
\hline 12 & & 0.112 \\
\hline
\end{tabular}

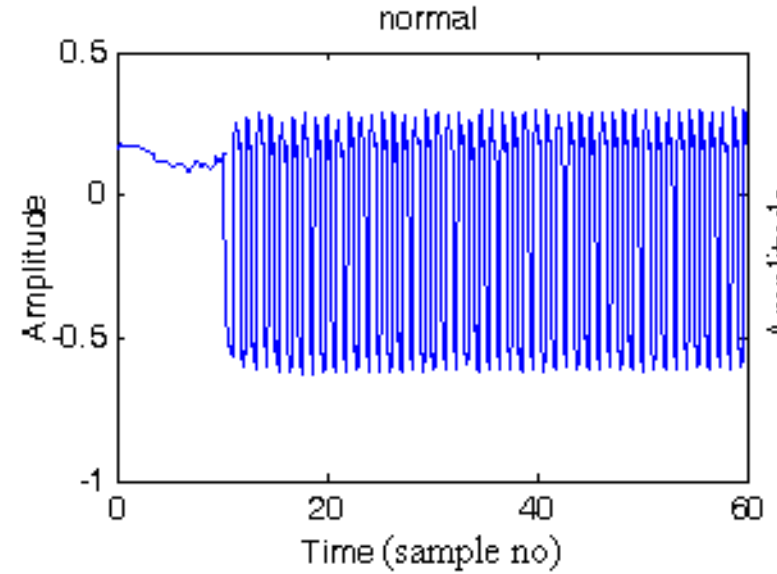

a)

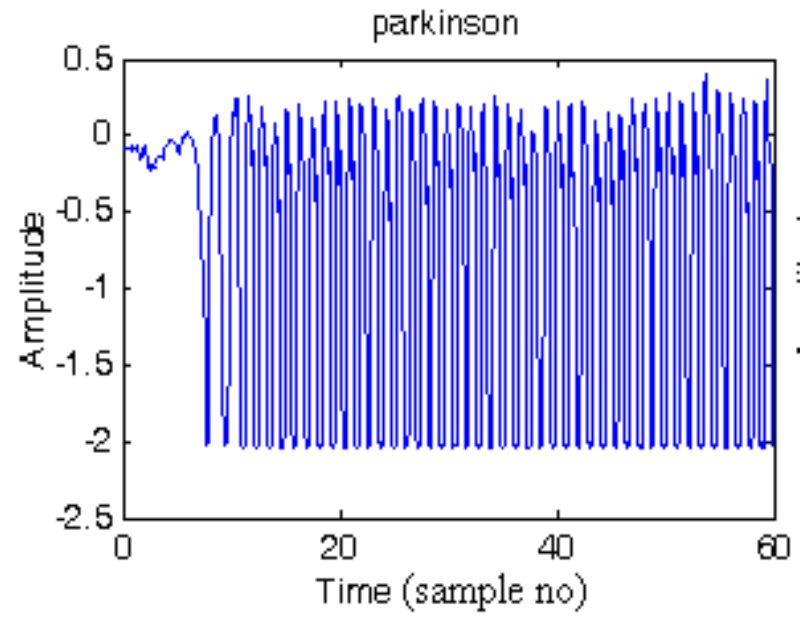

c)

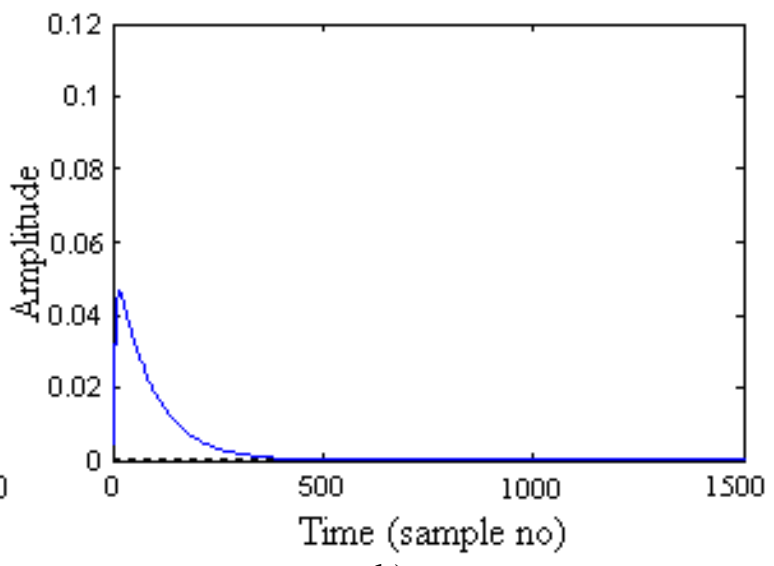

b)

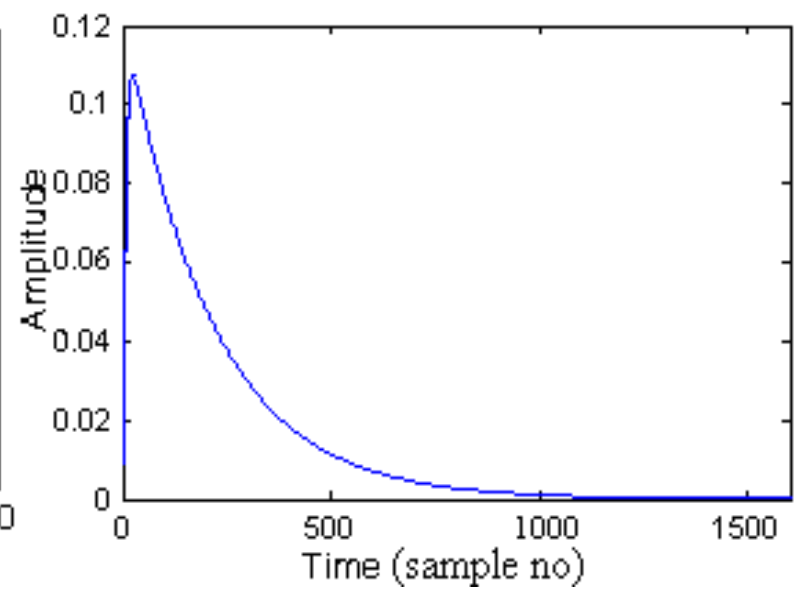

d) 


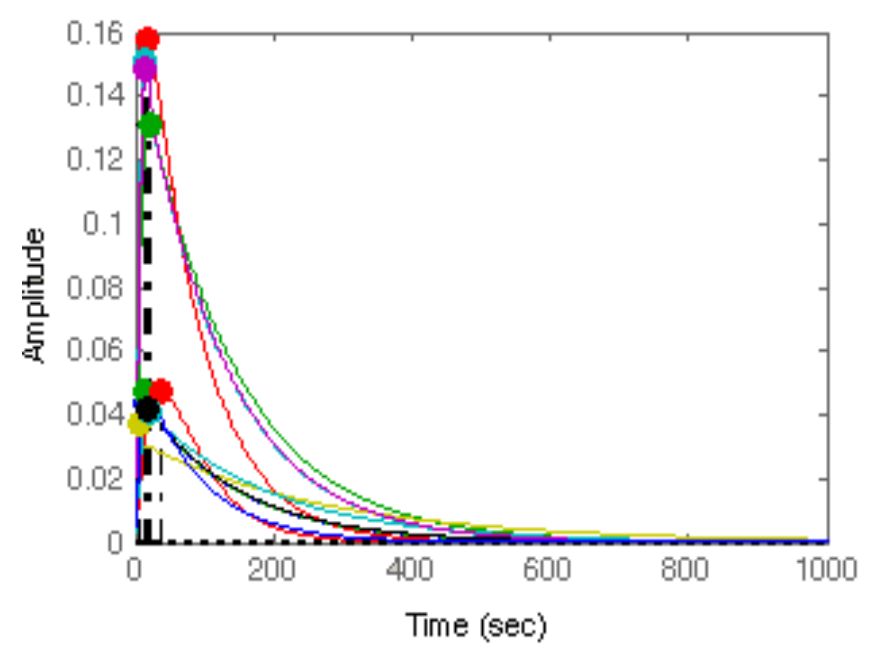

e)

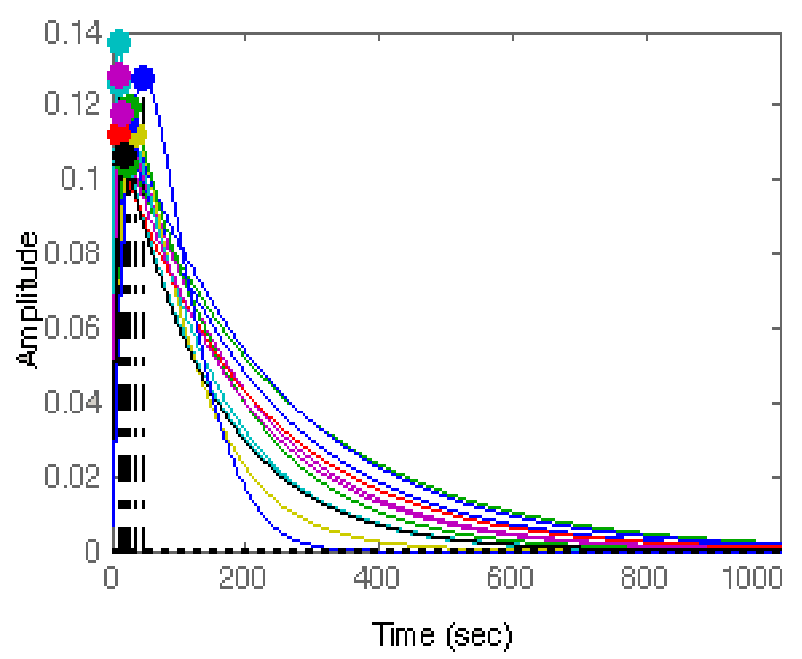

f)

Figure 3. a) Gait markers taken from normal individual

b) impulse model

c) Gait markers taken from Parkinson's patient

d) Parkinson's patient impulse model

e) Impulse responses of gait markers of 11 normal

f) Impulse responses of gait markers of $\mathrm{d} 12$ Parkinson's patients

\section{Conclusions}

The peak values of the impulse models of normal gait markers are collected in two groups. Whilst the first group typically has values in the region of 0.04 , the second group takes values in the region of $0.13,0.14$ and 0.15 .

The peak values of impulse models of Parkinson patients' gait markers are differentiated from normal markers, with values in the region of 0.11 and 0.12 (Figure $3 \mathrm{e}$ ),f) and Table 1).

\section{References}

[1] Beal, M.F., Lang, A.E., Ludolph, A.C., Neurodegenerative Diseases: Neurobiology,

Pathogenesis and Therapeutics, Cambridge University Pres, . London, UK, 2005.

[2] Galimberti, D., Scarpini, E., Neurodegenerative Diseases: Clinical Aspects, Molecular Genetics and Biomarkers, Springer London, 2014.

[3] Yalcın S., Ozaras N., Gait Analysis, Avrupa Publications, Istanbul, 2001 (inTurkish).

[4] Whittle, M.W., Gait Analysis: An Introduction, Butterworth-Heinemann, Waltham, 2007.

[5] Gabel, M. , Gilad-Bachrach, R., Renshaw, E., Schuster, A. (2012), Full Body Gait Analysis with Kinect, Engineering in Medicine and Biology Society (EMBC), Annual International Conference of the IEEE, San Diego, CA

[6] Hausdorff, J.M., Mitchell, S.L., Firtion, R., Peng, C.K., Cudkowicz, M.E., Wei, J.Y., Goldberger. A.L. (1997). Altered fractal dynamics of gait: reduced stride-interval correlations with aging and Huntington's disease. J. Applied Physiology, 82, 262-269. 
[7] Hausdorff, J.M., Lertratanakul, A., Cudkowicz, M.E., Peterson, A.L., Kaliton, D., Goldberger, A.L. (2000). Dynamic markers of altered gait rhythm in amyotrophic lateral sclerosis. J. Applied Physiology, 88, 2045-2053.

[8] Budzianowska, A, Honczarenko, K. (2008). Assessment of rest tremor in Parkinson's disease, Polish J. Neurol. Neurosurg., 42, $12-21$.

[9] Weiner, W.J., Shulman, L.M., Lang, A.E., Parkinson's Disease: A Complete Guide for Patients and Families, The Johns Hopkins University Pres, Baltimore, 2006.

[10] Pahwa, R., Lyons, K., Treatment Decisions in Parkinson's Disease, Oxford University Pres, Northants, 2010.

[11] Watanabe, K., Hokari, M. (2006). Kinematical analysis and measurement of sports form, IEEE Trans. Syst. Man Cybern. Part A, 36, 549-557.

[12] Kimmeskamp, S., Hennig, E.M. (2001). Heel to toe motion characteristics in Parkinson patients during free walking. Clin. Biomech. ;16, 806-812.

[13] Walter, E., Pronzato, E., Identijication of Parametric Models from experimental data, Springer, Londra, 1997.

[14] Tsuruoka, M., Tsuruoka, Y., Shibasaki, R., Yasuoka, Y., Murai, S. (2004). Analysis of impulse response on walking stability using accelerometers, Engineering in Medicine and Biology Society, 2, $4924-4927$.

[15] Akgun, O., Demir, H., Akan, A, (2008). Detection of ALS Disease Using AR Models of Gait Dynamics, Electrical, Electronic and Computer Engineering Symposium (ELECO), Bursa Turkey.

[16] Tsuruoka, M., Tsuruoka, Y., Shibasaki, R., Yasuoka, Y., Murai, S, (2001). Analysis of impulse response on walking stability by a synchronized system of two accelerometers. Computer-Based Medical Systems, 348 - 353.

[17] Tsuruoka, M., Tsuruoka, Y., Shibasaki, R., Yasuoka, Y., Murai, S. (1999). Spectral analysis of human movement stability using time series data in medicine. Computer-Based Medical Systems, $190-195$. 